Proceedings of the 2002 IEEE/RSJ

intl. Conference on Intelligent Robots and Systems

EPFL, Lausanne, Switzerland • October 2002

\title{
High resolution terrain mapping with an autonomous blimp
}

\author{
Simon Lacroix and Il-Kyun Jung \\ LAAS/CNRS \\ 7, av. du Colonel Roche \\ F-31077 Toulouse Cedex 4 - France \\ First-Name.Lastname@laas.fr
}

\begin{abstract}
This paper presents the current status of the development of our autonomous blimp project. Details are given on the hardware setup, which is currently almost operational. Some first experimental results on terrain mapping terrain mapping with low altitude stereo imagery are presented and discussed. The approach involves the intregration of several algorithms: stereovision, interest point matching, motion estimation and digital elevation map building.
\end{abstract}

\section{Introduction}

Our long term objectives in field robotics is to tackle the various issues raised by the deployment of heterogeneous autonomous systems, in the context of exploration, surveillance and intervention missions. Within such contexts, aerial robots will undoubtly play a growing role in the near future, not only during the mission preparation phase (in which drones can gather environment informations for instance), but also on-line, during the mission execution. Aerial robots can then provide the rovers with telecommunications support, as well as with up-to-date informations on the environment. They can also localize the rovers as they evolve within this environment, and even achieve by themselves some of the mission goals.

In the context of our field robotics project, we recently initiated the development of an autonomous blimp. While not being unconditional promoters of blimps and airships, we are convinced that the ever on-going developments in a wide spectrum of technologies, ranging from actuators, sensors and computing devices to energy and material will ensure lighter than air machines a promising future. There is undoubtly a regain of interest in this domain, as shown by the recent industrial developments on heavy loads transportation projects (such as CargoLifter and the ATG Skycats ${ }^{1}$, not to mention various other prospective transportation projects), and on stratospheric telecommunication platforms. As for smallsize unmanned radio-controlled models, which size is of

\footnotetext{
${ }^{1}$ up-to-date informations on these projects at www.cargolifter.com and www airship.com
}

the order of a few tens of cubic meters, their properties makes them an very suitable support to develop heterogeneous air/ground robotics systems: they are easy to operate, they can safely fly at very low altitudes (down to a few meters), and especially their dynamics is comparable with the ground rovers dynamics, as they can hover a long time over a particular area, while being able to fly at several tens of kilometers per hour. Their main and sole enemy is the wind (see [5] for a detailed and convincing review of the pros and cons of small size airships with regards to helicopters and planes). Finally, let's also note that some specific applications of unmanned blimps are more and more seriously considered throughout the world, from planetary exploration to military applications, as shown by numerous contributions in the AIAA Lighter Than Air conferences for instance [1].

The first mentions of the development of unmanned autonomous blimps can be found in the literature of the late 80 's, but it's only recently that various projects have reached effective achievements. One of the nicest $\mathrm{Au}$ rora, a project held at the Information Technology Institute of Campinas, Brazil, mainly devoted to flight control $[3,2]$, but within which other issues are also considered [4]. Interesting projects are aiso under development at the university of Virginia [10], at the university of Stuttgart $[11,8]$ and in various other labs.

Besides long-term developments related to the coordination and cooperation of heterogeneous air/ground robots, our research work on autonomous blimps is currently twofold: we concentrate on the definition of blimp trajectory control laws, and on environment modeling issues using low altitude imagery. For that purpose, we acquired in the end of 2001 a 15 cubic meter airship, and defined the necessary on-board equipment.

This paper presents our approach to terrain mapping with low altitude stereoscopic imagery. After a presentation of the experimental platform, section 3 describes the individual algorithms required by the terrain mapping: stereovision, motion estimation between stereo frames, and digital elevation map building. Section 4 present some first results. 


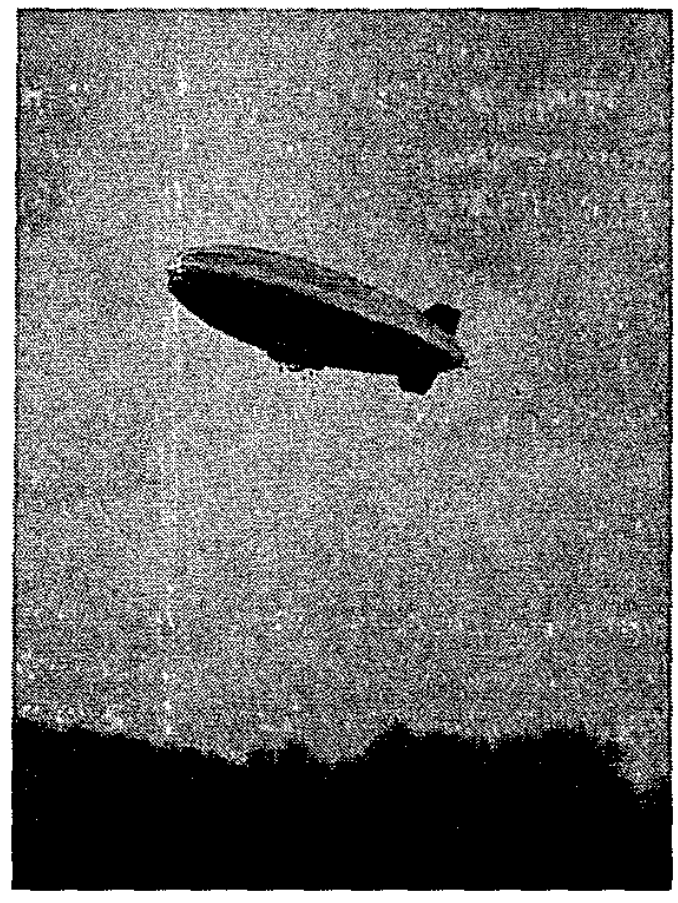

Figure 1: Our AS500 during its first test flight in November 2001 (no on-board instrument were installed at that time)

\section{The experimental platform}

\subsection{An Airspeed Airship AS-500}

Various radio-controlled blimps are built throughout the world. After one year of investigations, we decided to acquire and AS-500 model from the english company Airspeed Airship ${ }^{2}$. Criteria for this choice were the size of the blimp (which we wanted to be rather small, for the ease of deployment and stocking), its available payload and its possible operation modes.

Nominal specifications. According to the constructor, the technical characteristics of the original AS- 500 model are the following:

- $7.8 \mathrm{~m}$ long, $1.80 \mathrm{~m}$ max diameter, giving a volume of about $15.0 \mathrm{~m}^{3}$, and a fitness ratio of 4.25 .

- Vectorized thrust $\left(100^{\circ}\right.$ range), with two $7.5 \mathrm{~cm}^{3} \mathrm{en}$ gines, allowing speeds up to $45 \mathrm{~km} / \mathrm{h}$, possible control in wind gusts up to $25 \mathrm{~km} / \mathrm{h}$, and an endurance of 40 minutes with a $1.0 \mathrm{~kg}$ fuel load.

- The hull is made of welded mylar, and equipped with 4 control rudders in a " $x$ shape" configuration.

\footnotetext{
${ }^{2}$ The company's homepage is www.airship.demon.co.uk/airspeed.html - the platform used in the Aurora project is an AS-800, a similar bigger model.
}

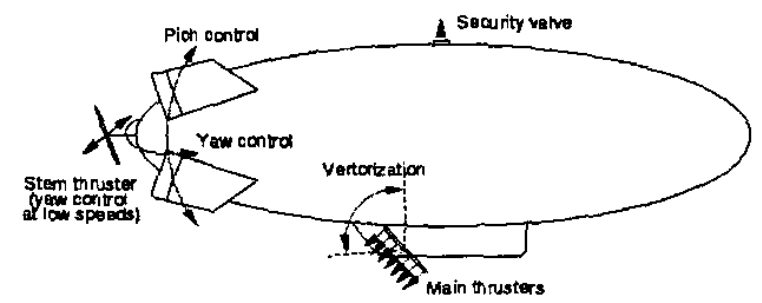

Figure 2: The various control parameters of our AS-500

A ballonet fed with air captured at the rear of the propellers maintains a constant hull pressure, and a radio-controlled valve on top of the hull can release helium when the temperature is getting higher.

- The static payload of the AS-500 (i.e. to reach the equilibrium) is $3.5 \mathrm{~kg}$, and the blimp must always be overweighted of 1.5 to $2.0 \mathrm{~kg}$ in flight: the maximal available payload is therefore about $5 \mathrm{~kg}$.

Specific modifications. In collaboration with Airspeed Airships, we specified the following modifications for our purpose:

- Electric motors: to have a finer controllability, we preferred to opt for electric motors. They do not weigh more than fuel engines, but are less powerful, thus reducing the maximum reachable speed and the possibility to fly in wind gusts. Their main drawback is the weight of the required batteries, which considerably reduces the available payload. We will however see in section 2.3 that thanks to lighter batteries and possible alternative energy sources, this drawback can be overcomed.

- Stern thruster: the rudder control surfaces require a certain speed to allow changes in both the altitude and orientation of the blimp. In order to have the possibility to maneuver the blimp while hovering, we choose to add a stern thruster, that gives the possibility to control the yaw angle.

The various control parameters of the blimp are sketched in figure 2. With the specified configuration, we made a first test flight, in order to check the available payload. It turned out that the static payload to reach equilibrium decreased down to $1.5 \mathrm{~kg}$, due to the batteries weight. We could successfully fly and maneuver the blimp with $1.3 \mathrm{~kg}$ overweight (the whole available payload is therefore $2.8 \mathrm{~kg}$ ), but the flight autonomy is not longer than 15 minutes with the batteries provided.

\subsection{On-board equipment}

To transform the blimp from a radio-controlled machine to a robot, we equipped it with a set of proprioceptive and 
exteroceptive sensors, and with computing and communications capabilities.

Stereovision. One of the advantage of having a big platform is that it can carry a wide base stereo bench, thus having the possibility to directly gather $3 \mathrm{D}$ data on the overflown ground. We adapted 2 high resolution digital $\mathrm{B} \& \mathrm{~W}$ cameras on a rigid $2 m$ carbon profile that traverses the $1.4 m$ long AS-500 gondola.

Blimp state observation. In order to tackle the flight control problem (and also to ease the development of mapping algorithms), we added the following sensors: a differential GPS receiver, a fluxgate compass, that also provide the blimp pitch and roll angles, and a wind sensor (sonar transducer technology), that measures the speed and orientation of the relative wind in the longitudinal plane.

CPU. We opted for a Matrox 4Sight board: it is an EBX form factor PC motherboard, endowed with all the necessary communication ports (100base-T ethernet, two USB ports, two RS232 ports, 16 TTLs, and especially 3 firewire ports). The board comprises a PCI04 slot, on which we added four more RS232 ports and a PCMCIA interface to host a light $11 \mathrm{Mbits} / \mathrm{s}$ ethernet modem card. Thanks to the $566 \mathrm{Mhz}$ Celeron processor, vision algorithms will eventually run on-board the CPU.

Actuator control. The control surfaces and motor servos of the blimp are usual PWM controlled modelist devices. We use a single chip $^{3}$ that can generate up to 8 sustained PWM signals from a RS232 input to control the actuators. For safety reasons, it is essential that an operator can retrieve the blimp control from the ground with the radio, at anytime: this is done thanks to a radio controlled switch, that cuts the PWM signals coming out from the chip.

The whole hardware architecture is sketched in figure 3 , and some specifications of the various devices are summarized in table 2.2. The total equipment weight is $1.520 \mathrm{~kg}$, which leaves only $1.300 \mathrm{~kg}$ for the various mechanical parts, wires and batteries that must provide the necessary additional $40 \mathrm{~W}$.

\footnotetext{
${ }^{3}$ A FerretTronics FT629
}

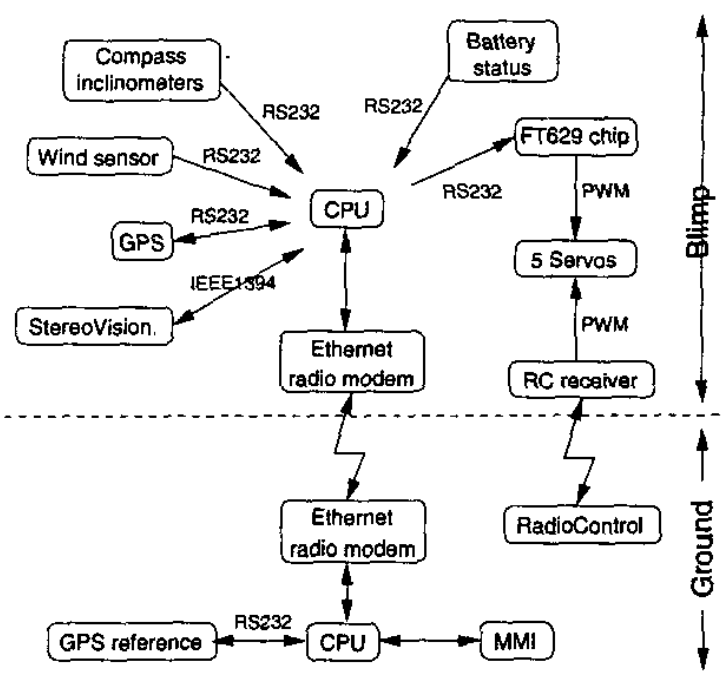

Figure 3: System hardware architecture

\begin{tabular}{|l|c|c|c|}
\hline $\begin{array}{l}\text { Device } \\
\begin{array}{l}\text { Compass } \\
\text { Inclinome- } \\
\text { ter }\end{array}\end{array}$ & Weight & Energy & Specs/ comments \\
\hline Wind sensor & $240 \mathrm{~g}$ & $0.25 \mathrm{~W} @ 12 \mathrm{~V}$ & $\begin{array}{c}\text { yaw }: 1.0^{\circ} \mathrm{RMS} ; \\
\text { Pitch/Roll }: 0.2^{\circ} \\
\text { RMS }\end{array}$ \\
\hline GPS & $110 \mathrm{~g}$ & $2.0 \mathrm{~W} @ 5 \mathrm{~V}$ & $\begin{array}{c}\text { Acc. }: 0.05 \mathrm{~m} / \mathrm{s}, 1.5^{\circ} \\
0.05 \mathrm{~m} / \mathrm{s}\end{array}$ \\
\hline $\begin{array}{l}\text { Stereo Cam- } \\
\text { eras }\end{array}$ & $2 \times 80 \mathrm{~g}$ & $\begin{array}{c}2 \times \\
2 \mathrm{~W} @ 12 \mathrm{~V}\end{array}$ & $\begin{array}{c}1280 \times 1024 \mathrm{~B} \& \mathrm{~W} \\
\text { images, } 10 \text { bits pixels }\end{array}$ \\
\hline CPU & $850 \mathrm{~g}$ & $30 \mathrm{~W} @ 12 \mathrm{~V}$ & $\begin{array}{c}\text { Including } 2 \mathrm{PC} 104 \\
\text { boards, 20 Go HD, } \\
\text { modem }\end{array}$ \\
\hline Lenses & $2 \times 65 \mathrm{~g}$ & & $\begin{array}{c}36^{\circ} \times 45^{\circ} \text { field of } \\
\text { view }\end{array}$ \\
\hline Total & $1.520 \mathrm{~kg}$ & $40 \mathrm{~W} @ 12 \mathrm{~V}$ & \\
\hline
\end{tabular}

Current developments. The various blimp state estimation sensors mentioned above provide the sufficient informations to tackle flight control in aerostatic mode (i.e. hovering or flying at very low speeds). But to control aerodynamic modes, the pitch and yaw rate informations are necessary. For that purpose, we are considering the addition of two solid-state rate gyros for that purpose. Also, the altitude estimate provided by the GPS receiver is not precise enough to safely servo the taking-off and landing phases: for that purpose, we will add a sonar telemeter. Fortunately, the main drawback of sonars in robotics, i.e. their wide perception cone which make their data interpretation so tedious, will turn into an advantage in our case, as there will be no need to mechanically stabilize it along the vertical. Finally, we are still investigating for 3D wind sensors (Pitot tubes are unfortunately not sensitive enough at the low blimp speeds). 

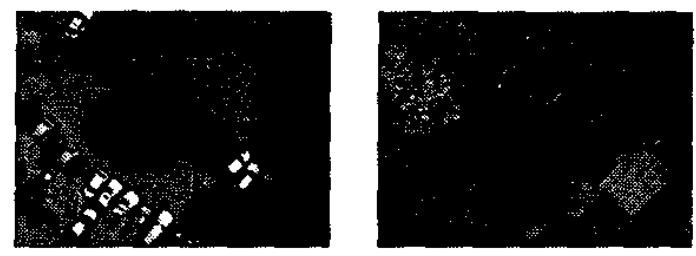

Figure 4: Two examples of images taken from the balloon.

\subsection{Energy management}

Energy is a critical issue for any flying device, mainly for safety considerations. In our case, we also would like to have more autonomy, while satisfying the maximal payload constraint.

Our AS-500 was originally equipped with 3 sets of $\mathrm{NiCd}$ batteries: one for the radio receiver and the servos $(4.8 \mathrm{~V}$, $1.2 \mathrm{Ah}, 0.140 \mathrm{~kg})$, one for the stern thruster $(7.2 \mathrm{~V}, 2.0 \mathrm{Ah}$, $0.350 \mathrm{~kg})$ and one for the main thrusters $(14.4 v, 5.0 \mathrm{Ah}$, $1.880 \mathrm{~kg}$ ).

We decided to replace the two thruster batteries with Lithium/Ion batteries, that have a much more interesting power/weight ratio (about 2.5 times better, which respectively saves $1.200 \mathrm{~kg}$ and $0.200 \mathrm{~kg}$ weight for the main and stern thrusters); and to feed the instruments with an other separate set of batteries. In total, the blimp now has 4 power sources, each of them being critical for its operation. So each battery is managed by a Maxim MAX1648 chip, that allows both the "intelligent" charge of the battery and the dispatching of status informations to the CPU via a multiplexed serial link. The four charging modules are linked to a single connector, on which a power source is plugged while the blimp is on the ground (which allows booting and debugging without any power loss), and on which a rescue set of non rechargeable Lithium/Ion batteries is plugged in flight (an additional weight of only $0.200 \mathrm{~kg}$ to deliver $5.0 \mathrm{Ah} @ 15 \mathrm{~V}$ ). This very flexible structure also allows the future use of an alternate power source on flight, such as a Stirling engine of a fuel cell.

\section{High resolution terrain mapping}

\subsection{Experimental Setup}

We acquired about 3000 stereo frames at altitudes ranging from 20 to 30 meters. The surface over which the balloon flew contains elements of various natures: rocky areas, grass areas, fences, tall trees and bushes, small buildings and a parking lot (figure 4).

\subsection{Stereovision}

Our stereovision algorithm is a classical pixel correlation algorithm [6]. A dense disparity image is produced from a pair of images thanks to a correlation-based pixel matching algorithm (we use either the ZNCC criteria or the census matching criteria), false matches are avoided thanks to a reverse correlation. With good quality images, the reverse correlation phase is enough to remove the few false matches that occur. Figure 5 presents some results of the algorithm on various image pairs.
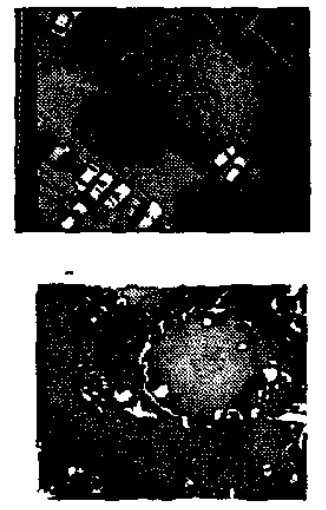
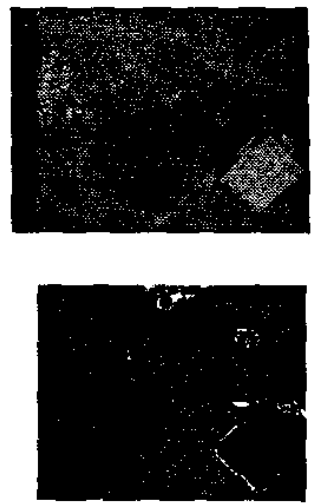

Figure 5: Results of the stereovision algorithm on two example images. Original images where non-matched pixels are white are on the top line, disparity images are on the bottom line.

\subsection{Building a DEM}

The main difficulty to build a digital elevation map comes from the uncertainties on the 3D input data, that can hardly be propagated throughout the computations and represented in the grid structure. In the 3D data, the range (depth) coordinate is the most unprecise, especially in the case of stereovision, where this uncertainty grows quadratically with the distance. But the problem is well conditioned when using aerial images looking downwards. Not only the data resolution on the ground is regular, but aiso the uncertainties on the 3D data "fits" well a representation of the uncertainties in the digital map: the uncertainties in the data can be fairly well estimated by a standard deviation on the cell elevation.

Considering these properties of the data, our algorithm to build a DEM therefore comes down to computing the elevation of the cell by averaging the elevations of the 3D points that are projected in the cells. The standard deviation on the elevation is also straightforwardly computed, and since to each 3D point is associated a luminance value, it is also possible to compute a mean luminance value for each map cell (figure 6).

\subsection{Motion Estimation}

In our experiments, the balloon positioning device have not beenn used: one must determine the relative positions of the system between successive stereo frames in order to build a global digital elevation map. For that purpose, we adapted a motion estimation algorithm we initially developed for ground rovers (sketched in figure 7 - more details can be found in [9]). 

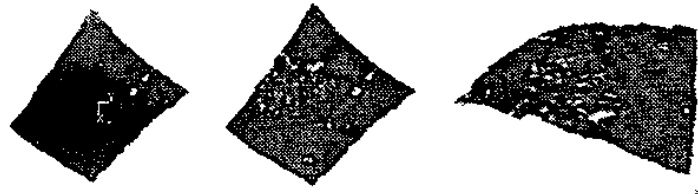

Figure 6: A digital elevation map computed with a single stereovision image. From left to right: top view of the elevations coded in grey levels, top view of the luminance values stored in the DEM, and $3 \mathrm{D}$ view. The horizontal plane of the DEM is here defined orthogonally to the view axis of the stereovision image considered.

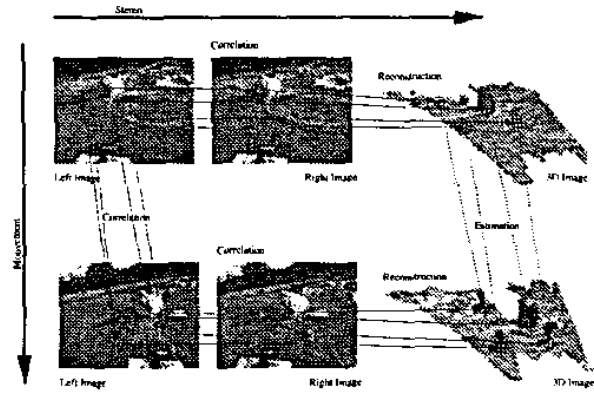

Figure 7: Principle of the visual motion estimation technique. The steps of stereovision go from left to right, time goes from top to bottom. Given a stereovision image pair, a set of pixels are selected on the left image. They are tracked and matched in the new stereovision frame: this produces 3D points associations (right - the $3 \mathrm{D}$ images are represented as DEMs for readability purposes), from which the motion is estimated.

But when applied with a ground rover, the tracking phase is initiated using a first estimate of the motion provided by odometry. This is not possible with the balloon images: no initial motion estimate being provided, there is no way to focus the search area in the images to track the pixels.

This phase is therefore replaced by an algorithm that matches interest points detected on a pair of grey level images taken from arbitrary points of view. First matching hypotheses are generated using a similarity measure of the interest points. Hypotheses are confirmed using local groups of interest points: group matches are based on a measure defined on an affine transformation estimate and a on correlation coefficient computed on the intensity of the interest points that are consistent with the estimated affine transformation. Once a reliable match has been determined for a given interest point and the corresponding local group, new group matches are found by propagating the estimated affine transformation (more details on this algorithm can be found in [7]).

The algorithm provides dense matches and is very robust to outliers, i.e. interest points generated by noise or present in only one image because of occlusions or non overlap. Figure 8 shows some results of the inter- est points matching algorithm on some images acquired with the balloon. The algorithm is extremely robust: it has been tested with hundreds of images of various $3 \mathrm{D}$ scenes, taken in various conditions and environments.
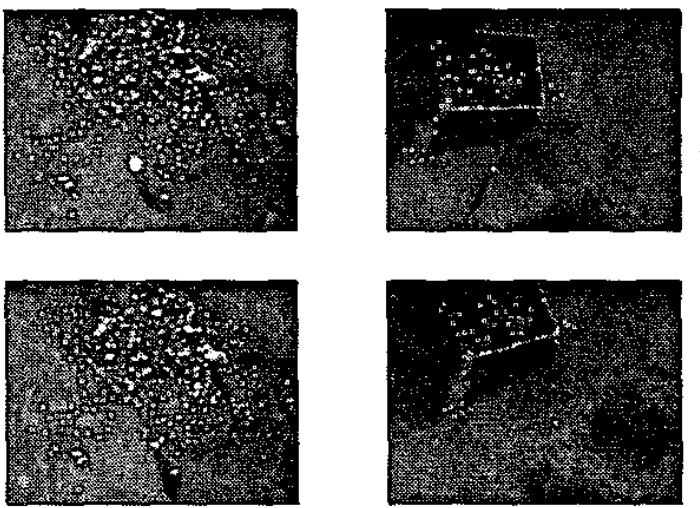

Figure 8: Three results of the interest point matching algorithm. The white squares indicate the interest points that were matched. Note that the viewpoint changes in the middle and right images are quite important: nevertheless, no erroneous matches were established.

\section{First results}

Thanks to these three algorithms (stereovision, motion estimation and map building), we could build various digital elevations maps integrating several tens of stereo images $^{4}$.

Figure 9 and 10 presents a digital elevation map built with about 120 stereovision pairs, covering about $1500 \mathrm{~m}^{2}$, with a cell resolution of $5 \times 5 \mathrm{~cm}^{2}$. The trajectory executed by the blimp, which could be recovered using the localization algorithm, is an about $100 \mathrm{~m}$ long loop. The last images overlaps the first images, and no discrepancies can be observed in the final model on this area: the localization algorithm gave here an extremely precise position estimate, with a final error of the order a map cell size, i.e. about $0.1 \%$. Figure 11 shows the map built with all the images of the third trajectory: the position estimation in this latter case drifted of about $1 \%$ (see the discrepancies highlighted by blue ellipses).

All the images were processed off-line, but the computations times are compatible with an on-line implementation. Most of the time being consumed by stereovision and interest points matching, which both take about one second on an Ultra-10 Sparc CPU (an optimized compilation on-board the Celeron of the AS-500 should dramatically speed up these performances).

\footnotetext{
${ }^{4}$ In the absence of any attitude estimation device on-board the blimp, the plane of the DEM is defined orthogonally to the view axis of the first stereovision pair considered.
} 

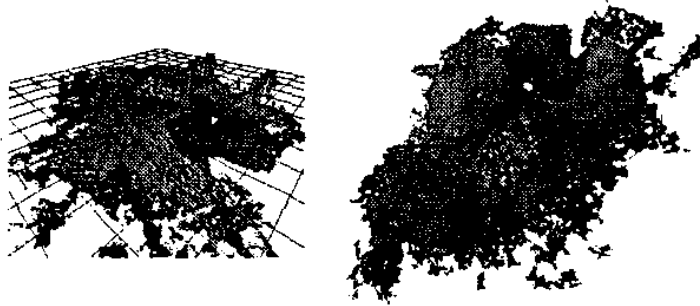

Figure 9: Digital elevation map produced with about 120 stereovision images. Left: 3D model, in which some peaks are artifacts due to the presence of the moving operator. Right: a top view of this model, which is actually an ortho-image of the terrain. The "vertical" projection of the blimp positions are shown as small black frames in this image.
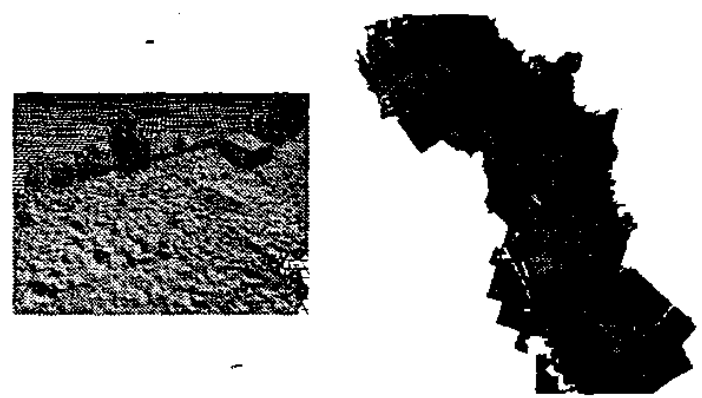

Figure 10: An other example produced with about 120 stereovision images. The 3D view is a close up of the bottom of the orthoimage.

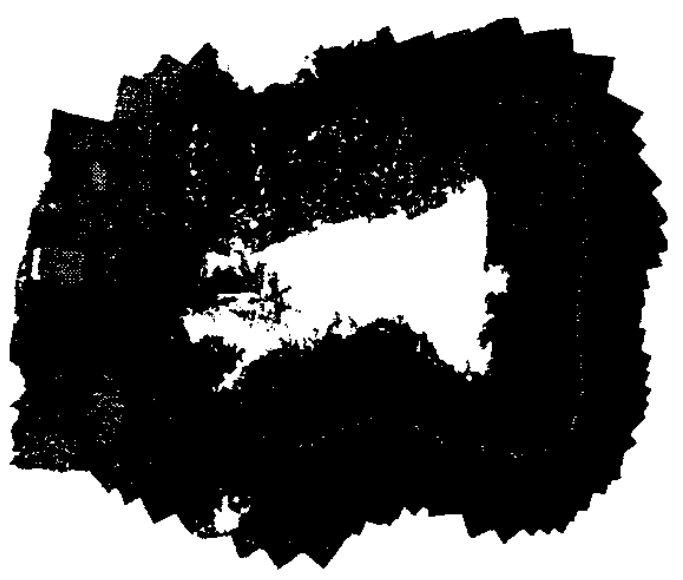

Figure 11: An other digital elevation map produced with about 200 stereovision images. The overall position estimation error can be seen on top left of the image, where the first and the last images overlap (blue ellipse). The absolute translation error is here about $1 \mathrm{~m}$ at the end of the trajectory.

\section{Discussion}

The localization method we used here (which we refer to as "optical odometry") eventually drifts, as errors are cumulated over the distance traveled. Current work concen- trates on the development of a "SLAM-based" approach, in which some of the interest points would be used as landmarks.

From a more prospective points of view, we are investigating the possibility to integrate various sources of aerial informations in the DEM, including data acquired by a ground rover. The latter problem is a very important problem, as it is a basis upon which future collaborative scenarios can be built among air/ground robots.

\section{References}

[1] AIAA. Lighter-Than-Air Convention and Exhibition, Akron, OH (USA), July 2001.

[2] J.R. Azinheira, E. de Paiva, J. Ramos, S.S, Bueno, M. Bergerman, and S.B.V. Gomes. Extended dynamic model for aurora robotic airship. In 14th AIAA Lighter-Than-Air Conference and Exhibition, Akron, Ohio (USA), July 2001.

[3] E.C. de Carneiro, S.S. Bueno, and M. Bergerman. A robust pitch attitude controller for aurora's semin-autonomous robotics airship. In 13th AIAA Lighter-than-air Systems Technology Conference, Norfolk, Va. (USA), pages 141148, July 1999.

[4] A. Elfes, M. Bergermann, and S. Bueno. The potential of robotic airships for planetary exploration. In 10th International Conference on Advanced Robotics, Budapest (Hungary), pages 131-138, Aug. 2001.

[5] A. Elfes, S.S. Bueno, M. Bergerman, J.G. Ramos, and S.B Varella Gomes. Project.AURORA: development of an autonomous unmanned remote monitoring robotic airship. Journal of the Brazilian Computer Society, 4(3):7078, April 1998.

[6] O. Faugeras, T. Vieville, E. Theron, J. Vuillemin, B. Hotz, Z. Zhang, L. Moll, P. Bertin, H. Mathieu, P. Fua, G. Berry, and C. Proy. Real-time correlation-based stereo : algorithm, implementations and application. Technical Report RR 2013, INRIA, August 1993.

[7] I-K. Jung and S. Lacroix. A robust interest point matching algorithm. In 8th International Conference on Computer Vision, Vancouver (Canada), July 2001.

[8] P. Kungl, M. Schlenker, and B. Kröplin. Research and testing activities with the solar powered airship lotte within the scope of the airship research group at the university of stuttgart. In 14th AIAA Lighter-Than-Air Conference and Exhibition, Akron, Ohio (USA), July 2001.

[9] A. Mallet, S. Lacroix, and L. Gallo. Position estimation in outdoor environments using pixel tracking and stereovision. In IEEE International Conference on Robotics and Automation, San Francisco, Ca (USA), pages 3519-3524, April 2000.

[10] A. Turner. Development of a semi-autonomous control system for the uva solar airship aztec. In 3rd International Airship Convention and Exhibition. Friedrichshafen (Germany), July 200.

[11] D.A. Wimmer and K.H. Well. Instrumentation, identification and control of airship lotte. In 14th AIAA LighterThan-Air Conference and Exhibition, Akron, Ohio (USA), July 2001. 